\title{
THE INVISCID LIMIT FOR TWO-DIMENSIONAL INCOMPRESSIBLE FLUIDS WITH UNBOUNDED VORTICITY
}

\author{
JAMES P. KELLIHER
}

\begin{abstract}
In [C2], Chemin shows that solutions of the Navier-Stokes equations in $\mathbb{R}^{2}$ for an incompressible fluid whose initial vorticity lies in $L^{2} \cap L^{\infty}$ converge in the zero-viscosity limit in the $L^{2}$-norm to a solution of the Euler equations, convergence being uniform over any finite time interval. In [Y2], Yudovich assumes an initial vorticity lying in $L^{p}$ for all $p \geq p_{0}$, and establishes the uniqueness of solutions to the Euler equations for an incompressible fluid in a bounded domain of $\mathbb{R}^{n}$, assuming a particular bound on the growth of the $L^{p}$-norm of the initial vorticity as $p$ grows large. We combine these two approaches to establish, in $\mathbb{R}^{2}$, the uniqueness of solutions to the Euler equations and the same zero-viscosity convergence as Chemin, but under Yudovich's assumptions on the vorticity with $p_{0}=2$. The resulting bounded rate of convergence can be arbitrarily slow as a function of the viscosity $\nu$.
\end{abstract}

\section{Introduction}

The equations of motion governing an incompressible fluid with viscosity $\nu$ are the Navier-Stokes equations,

$$
\left(N S_{\nu}\right) \quad\left\{\begin{array}{c}
\partial_{t} v_{\nu}+v_{\nu} \cdot \nabla v_{\nu}-\nu \Delta v_{\nu}=-\nabla p_{\nu} \\
\operatorname{div} v_{\nu}=0 \\
\left.v_{\nu}\right|_{t=0}=v^{0}
\end{array}\right.
$$

These same equations with zero viscosity become the Euler equations:

$$
(E) \quad\left\{\begin{array}{c}
\partial_{t} v+v \cdot \nabla v=-\nabla p \\
\operatorname{div} v=0 \\
\left.v\right|_{t=0}=v^{0}
\end{array}\right.
$$

The question of whether a solution to $\left(N S_{\nu}\right)$ converges, by some measure, to a solution to $(E)$ as $\nu \rightarrow 0$ (the inviscid or zero-viscosity limit) has a long history. Temam has a discussion of this in Appendix III of [T]. See also Kato's remarks in $[\mathrm{K}]$. Briefly, convergence of smooth solutions in $\mathbb{R}^{n}$ is well understood. Much less is known about convergence of weak solutions in $\mathbb{R}^{n}$ or the convergence of solutions, weak or smooth, in a domain with boundaries.

We restrict our attention to fluids extending throughout $\mathbb{R}^{2}$, with the initial velocity belonging, for some real number $m$, to the space $E_{m}$ of [C2] and [C3].

Received June 26, 2003.

2000 Mathematics Subject Classification. Primary 76D05, 76C99.

Key words and phrases. Fluid mechanics, inviscid limit. 
A vector $v$ belongs to $E_{m}$ if it is divergence-free and can be written in the form $v=\sigma+v^{\prime}$, where $v^{\prime}$ is in $L^{2}\left(\mathbb{R}^{2}\right)$ and where $\sigma$ is a stationary vector field, meaning that $\sigma$ is of the form,

$$
\sigma=\left(-\frac{x^{2}}{r^{2}} \int_{0}^{r} \rho g(\rho) d \rho, \frac{x^{1}}{r^{2}} \int_{0}^{r} \rho g(\rho) d \rho\right)
$$

where $g$ is in $C_{0}^{\infty}(\mathbb{R} \backslash\{0\})$. $E_{m}$ is an affine space; fixing an origin, $\sigma$, in $E_{m}$ we can define a norm by $\left\|\sigma+v^{\prime}\right\|_{E_{m}}=\left\|v^{\prime}\right\|_{L^{2}}$. Convergence in $E_{m}$ is equivalent to convergence in the $L^{2}$-norm to a vector in $E_{m}$.

We use the notation $\omega(v)$, or just $\omega$ when $v$ is understood, for the vorticity of $v$, which equals $\partial_{1} v^{2}-\partial_{2} v^{1}$. The initial vorticity we denote by $\omega^{0}$.

The following is a fundamental result of Yudovich's ([Y1]), as adapted by Chemin in [C1] from bounded domains to all of $\mathbb{R}^{2}$ (see [C3]):

Theorem 1.1 (Yudovich's theorem). Let $v^{0}$ be in $E_{m}$, with $\omega^{0}$ belonging to $L^{a}\left(\mathbb{R}^{2}\right) \cap L^{\infty}\left(\mathbb{R}^{2}\right)$ for some $1<a<\infty$. Then there exists a unique solution $v$ of $(E)$ belonging to $C\left(\mathbb{R} ; E_{m}\right)$ such that $\omega(v)$ is in $L^{\infty}\left(\mathbb{R}^{3}\right) \cap L^{\infty}\left(\mathbb{R} ; L^{a}\left(\mathbb{R}^{2}\right)\right)$.

In [Y2], Yudovich, in the setting of a bounded domain in $\mathbb{R}^{n}$ with impermeable boundary, weakens the conditions on the initial vorticity in Theorem 1.1, allowing unbounded vorticity, and is still able to obtain uniqueness. (Similar results have been obtained by Serfati in [S].) Chemin shows in [C2] that with the assumptions on the initial data in Theorem 1.1 with $a=2$, solutions $\left(v_{\nu}\right)_{v>0}$ of $\left(N S_{\nu}\right)$ converge in the $L^{2}$-norm uniformly over a finite time interval as $\nu \rightarrow 0$ to the unique solution $v$ of $(E)$ given by Theorem 1.1. We establish the same convergence as Chemin, but with the initial vorticity of Yudovich.

To describe Yudovich's conditions on the initial vorticity, let $\phi:\left[p_{0}, \infty\right) \rightarrow \mathbb{R}^{+}$ be a continuous function, where $p_{0}>1$. We define two functions, $\beta_{\epsilon, M, \phi}: \mathbb{R}^{+} \rightarrow$ $\mathbb{R}^{+}$and $\beta_{M, \phi}: \mathbb{R}^{+} \rightarrow \mathbb{R}^{+}$, parameterized by $\epsilon$ in $\left(0,1 / p_{0}\right], M>0$, and $\phi$ :

$$
\begin{aligned}
& \beta_{\epsilon, M, \phi}(x)=M^{\epsilon} x^{1-\epsilon} \phi(1 / \epsilon), \\
& \beta_{M, \phi}(x)=\inf \left\{\beta_{\epsilon}(x): \epsilon \in\left(0,1 / p_{0}\right]\right\} .
\end{aligned}
$$

For brevity, we usually write $\beta_{\epsilon}$ for $\beta_{\epsilon, M, \phi}$ and $\beta$ for $\beta_{M, \phi}$, with the choices of $M$ and $\phi$ being understood.

For all $\epsilon$ in $\left(0,1 / p_{0}\right], \beta_{\epsilon}(x)$ is a monotonically increasing function continuous in $x$ and in $\epsilon$, with $\lim _{x \rightarrow 0^{+}} \beta_{\epsilon}(x)=0$. It follows that $\beta$ is a monotonically increasing continuous function and that $\lim _{x \rightarrow 0^{+}} \beta(x)=0$. Also, $\beta(x) \leq \beta_{\epsilon}(x)$ for all $\epsilon$ in $\left(0,1 / p_{0}\right]$ and $x \in \mathbb{R}^{+}$.

Definition 1.2. A continuous function $\theta:\left[p_{0}, \infty\right) \rightarrow \mathbb{R}^{+}$is called admissible if

$$
\int_{0}^{1} \frac{d s}{\beta_{M, \phi}(s)}=\infty
$$

where $\phi(p)=p \theta(p)$. This condition is independent of the choice of $M$. 
In Section 2 we give examples of admissible functions and discuss how our definition relates to the equivalent definition in [Y2].

Yudovich proves that for a bounded domain in $\mathbb{R}^{n}$ with impermeable boundary (which adds the condition to $(E)$ that the normal component of the velocity on the boundary is zero), if the $L^{p}$-norms of the initial vorticity are bounded by an admissible function $\theta$, then at most one solution to $(E)$ exists.

For our purposes, we define (weak) solutions to $(E)$ and $\left(N S_{\nu}\right)$ as follows:

Definition 1.3. A time-varying vector field $v: \mathbb{R} \times \mathbb{R}^{2} \rightarrow \mathbb{R}^{2}$ is a weak solution to $(E)$ or $\left(N S_{\nu}\right)$ if there exists a distribution $p$ such that $(E)$ or $\left(N S_{\nu}\right)$ hold in the sense of distributions and if, in addition,

(i) $v$ is in $L_{l o c}^{\infty}\left(\mathbb{R} ; E_{m}\right)$ for some real $m$, and

(ii) there exists a $p_{0}>1$ such that $\nabla v$ is in $L_{l o c}^{\infty}\left(\mathbb{R} ; L^{p}\left(\mathbb{R}^{2}\right)\right)$ for all $p$ in $\left[p_{0}, \infty\right)$.

We combine the techniques of Chemin and Yudovich to prove the following theorem:

Theorem 1.4. Let $v^{0}$ be in $E_{m}$ and assume that $\omega^{0}$ is in $L^{p}\left(\mathbb{R}^{2}\right)$ for all $p$ in $[2, \infty)$, with $\left\|\omega^{0}\right\|_{L^{p}} \leq \theta(p)$ for some admissible function $\theta$. Then:

(i) There exists a unique solution $v$ of $(E)$.

(ii) For all $\nu>0$, there exists a unique solution $v_{\nu}$ of $\left(N S_{\nu}\right)$.

(iii) $\left\|v_{\nu}(t)-v(t)\right\|_{L^{2}} \rightarrow 0$ in $L^{2}\left(\mathbb{R}^{2}\right)$ uniformly on $[0, T]$ as $\nu \rightarrow 0^{+}$.

We prove only the uniqueness statements of (i) and (ii), a proof of existence following from the bounds we obtain on the $L^{2}$-norm of the difference between two solutions, much as in the proof of Theorem 1.1. It is also true that $v$ and $v_{\nu}$ lie in $C\left(\mathbb{R} ; E_{m}\right)$ (after possibly changing their values on a set of measure zero), but we do not use this fact.

Given an initial velocity in $E_{m}$, there exists a unique solution in the sense of distributions to $\left(N S_{\nu}\right)$ in $C\left([0, T] ; E_{m}\right) \cap L^{2}\left([0, T] ; \dot{H}^{1}\right)$ for all $T>0$. This is essentially a classical result of Leray, which can be proved, for instance, by straightforward modifications of the proofs of Theorems 3.1 and 3.2 of Chapter 3 of [T]. Additional assumptions, such as those of Theorem 1.4, are required, however, to conclude that the velocity is in $L^{\infty}\left([0, T] ; \dot{H}^{1}\right)$, not just in $L^{2}\left([0, T] ; \dot{H}^{1}\right)$.

The rate of convergence in the inviscid limit is also of interest. Constantin and $\mathrm{Wu}$ in [CW1] show that the $L^{2}$-rate of convergence of the velocity for a vortex patch in $\mathbb{R}^{2}$ with smooth boundary is $O(\sqrt{\nu t})$ uniformly over any finite time interval, and remark that this same result holds when $\nabla v$ is in $L_{l o c}^{1}\left(\mathbb{R} ; L^{\infty}\left(\mathbb{R}^{2}\right)\right)$, where $v$ is the solution to $(E)$. Chemin in $[\mathrm{C} 2]$ gives essentially the same bound on the convergence rate as that in $[\mathrm{CW} 1]$, assuming that $v$ is in $L_{l o c}^{\infty}\left(\mathbb{R}^{+} ;\right.$Lip $)$, which implies the condition in $[\mathrm{CW} 1]$ that $\nabla v$ lie in $L_{l o c}^{1}\left(\mathbb{R} ; L^{\infty}\left(\mathbb{R}^{2}\right)\right)$.

Chemin goes on to establish bounds on the rate of convergence given initial vorticity in $L^{2} \cap L^{\infty}$, the bounded rate of convergence always being slower than $O(\sqrt{\nu})$, but approaching that order for small time intervals. The approach we take leads, in the special case of $L^{2} \cap L^{\infty}$, to the same bound on the rate of 
convergence as Chemin. In the general case of unbounded vorticity, however, the bounded rate of convergence can be arbitrarily slow.

In [CW2], Constantin and Wu consider an initial vorticity in $\mathbb{R}^{2}$ lying in the space $\mathbf{Y}$ of bounded, compactly supported functions. They also assume that the initial vorticity lies in certain Besov spaces, and establish convergence of the vorticity in every $L^{p}$-norm for $p \geq 2$, with the rate of convergence increasing with increasing $p$. In [CW3], the same authors consider statistical solutions of $\left(N S_{\nu}\right)$ and their inviscid limits, working again with the space $\mathbf{Y}$.

We also note that given the uniqueness of the solution to $(E)$ in $\mathbb{R}^{2}$ established in Theorem 1.4, the compactness argument on p. 131-133 of [L] would imply the strong convergence in (iii) of Theorem 1.4. A bound on the rate of convergence does not follow from that approach, however.

We use without proof the following theorem:

Theorem 1.5. Let $v$ be a solution to $\left(N S_{\nu}\right)$ or $(E)$ as defined in Definition 1.3, and let $\sigma$ be any stationary vector field in $E_{m}$. Then:

(i) $v-\sigma$ is in $L_{\text {loc }}^{\infty}\left(\mathbb{R} ; L^{2}\left(\mathbb{R}^{2}\right)\right.$ ) (i.e., the $L^{2}$-norm of $v-\sigma$ is bounded over any finite time interval), the norm being bounded over $\{\nu>0\}$;

(ii) $v$ is in $L_{\text {loc }}^{\infty}\left(\mathbb{R} ; L^{\infty}\left(\mathbb{R}^{2}\right)\right)$, the norm being bounded over $\{\nu>0\}$;

(iii) $\|\omega(t)\|_{L^{p}} \leq\left\|\omega^{0}\right\|_{L^{p}}$ for all $1 \leq p \leq \infty$;

(iv) there exists a constant $C$ such that for all $p \geq 2,\|\nabla v\|_{L^{p}} \leq C p\|\omega\|_{L^{p}}$ when $\omega$ is in $L^{p}$.

In Theorem 1.5, (i) comes from energy estimates, as does (ii) after decomposing $v-\sigma$ into high and low frequencies. Equality holds in (iii) for solutions to $(E)$, and (iv) is a result from harmonic analysis that applies to all divergence-free vector fields in $\mathbb{R}^{n}$.

We will also need Osgood's lemma, the proof of which can be found, for example, on p. 92 of [C3].

Lemma 1.6 (Osgood's lemma). Let $L$ be a measurable positive function and $\gamma$ a positive locally integrable function, each defined on the domain $\left[t_{0}, t_{1}\right]$. Let $\mu:[0, \infty) \rightarrow[0, \infty)$ be a continuous nondecreasing function, with $\mu(0)=0$. Let $a \geq 0$, and assume that for all $t$ in $\left[t_{0}, t_{1}\right]$,

$$
L(t) \leq a+\int_{t_{0}}^{t} \gamma(s) \mu(L(s)) d s .
$$

If $a>0$, then

$$
-\mathcal{M}(L(t))+\mathcal{M}(a) \leq \int_{t_{0}}^{t} \gamma(s) d s, \text { where } \mathcal{M}(x)=\int_{x}^{1} \frac{d s}{\mu(s)} .
$$

If $a=0$ and $\mathcal{M}(0)=\infty$, then $L \equiv 0$. 


\section{Yudovich's Unbounded Vorticity}

Definition 1.2 is equivalent to requiring that

$$
\psi(x):=\inf \left\{\left(x^{\epsilon} / \epsilon\right) \theta(1 / \epsilon): \epsilon \in\left(0,1 / p_{0}\right]\right\}
$$

satisfy

$$
\int_{1}^{\infty} \frac{d x}{x \psi(x)}=\infty
$$

which is essentially the same as the condition in [Y2]. The functions $\psi$ and $\beta$ are related by $\psi(x)=x \beta(1 / x)$ when $M=1$.

Choosing $\epsilon=1 / \ln x$ in Equation (2.1) shows that $\psi(x) \leq e(\ln x) \theta(\ln x)$ when $x \geq \exp \left(p_{0}\right)$. It follows that

$$
\int_{1}^{\infty} \frac{d x}{x \psi(x)} \geq \int_{e^{p_{0}}}^{\infty} \frac{d x}{e x(\ln x) \theta(\ln x)}=\frac{1}{e} \int_{p_{0}}^{\infty} \frac{d p}{p \theta(p)}
$$

For $\theta$ to be admissible, it is sufficient, though not necessary, that the final integral in Equation (2.2) be infinite. Thus we can say, as a rough measure only, that the $L^{p}$-norm of the initial vorticity can grow in $p$ only slightly faster than $\log p$ and still be handled by our approach. Such growth in the $L^{p_{-}}$norm arises, for example, from a point singularity of the type $\log \log (1 / x)$.

Define, as in [Y2], the sequence of admissible bounds on vorticity,

$$
\theta_{0}(p)=1, \theta_{1}(p)=\ln p, \ldots, \theta_{m}(p)=\ln p \cdot \ln \ln p \cdots \ln ^{m} p,
$$

where $\ln ^{m}$ is $\ln$ composed with itself $m$ times. These are each admissible since $\psi(x) \leq e(\ln x) \theta_{m}(\ln x)=e \theta_{m+1}(x)$, and a repeated change of variables shows that the final integral in Equation (2.2) is infinite for $\theta=\theta_{m}$.

\section{Proof of Theorem 1.4}

We take a unified approach to proving the three parts of Theorem 1.4. Let each of $\left(v_{\nu}\right)_{\nu>0}$ and $\left(v_{\nu}^{\prime}\right)_{\nu>0}$ be either a family of solutions to $\left(N S_{\nu}\right)$ parameterized by the viscosity $\nu$ or a single solution to $(E)$. In the latter case, the solution is independent of the value of $\nu$. All solutions in $\left(v_{\nu}\right)_{\nu>0}$ and $\left(v_{\nu}^{\prime}\right)_{\nu>0}$ share the same initial velocity $v^{0}$, which lies in $E_{m}$ and satisfies the vorticity bounds assumed in Theorem 1.4. Let

$$
w_{\nu}=v_{\nu}-v_{\nu}^{\prime} .
$$

Theorem 3.1. Under the assumptions of Theorem 1.4, for all $t \geq 0$,

$$
\int_{\mathbb{R}^{2}}\left|w_{\nu}(t, x)\right|^{2} d x \leq R \nu t+2 \int_{0}^{t} \int_{\mathbb{R}^{2}}\left|\nabla v_{\nu}^{\prime}(s, x)\right|\left|w_{\nu}(s, x)\right|^{2} d x d s .
$$

$R=0$ when $w_{\nu}$ is the difference between two solutions to $\left(N S_{\nu}\right)$ and when $w_{\nu}$ is the difference between two solutions to $(E) . R>0$ when $w_{\nu}$ is the difference between a solution to $\left(N S_{\nu}\right)$ and a solution to $(E)$.

Proof. See Section 6. 
Theorem 3.2. Let $f_{\nu}$ and $g_{\nu}$ be nonnegative measurable real functions on $[0, T] \times$ $\mathbb{R}^{2}$ parameterized by $\nu>0$ for some $T>0$. Assume that $f_{\nu}(t)$ is in $L^{1}\left(\mathbb{R}^{2}\right)$ for all $t \in[0, T]$ and $\nu>0$, and that

$$
\sup _{\nu>0}\left\{\left\|f_{\nu}\right\|_{L^{\infty}\left([0, T] \times \mathbb{R}^{2}\right)}\right\}<\infty .
$$

Assume that for some $p_{0}>1$ and some function $\phi$, where $\phi(p)=p \theta(p)$ for an admissible function $\theta$,

$$
\left\|g_{\nu}(t, \cdot)\right\|_{L^{p}\left(\mathbb{R}^{2}\right)} \leq \phi(p)
$$

for all $t$ in $[0, T]$ and $p \geq p_{0}$. Assume also that for some real constant $R$,

$$
L_{\nu}(t):=\int_{\mathbb{R}^{2}} f_{\nu}(t, x) d x \leq R \nu t+\int_{0}^{t} \int_{\mathbb{R}^{2}} g_{\nu}(s, x) f_{\nu}(s, x) d x d s .
$$

If $R=0$ then $L_{\nu} \equiv 0$.

If $R>0$ then $L_{\nu}(t) \rightarrow 0$ uniformly on $[0, T]$ as $\nu \rightarrow 0^{+}$.

Proof. See Section 4.

Proof of Theorem 1.4. Fix a $T>0$ and let $f_{\nu}=\left|w_{\nu}\right|^{2}, g_{\nu}=2\left|\nabla v_{\nu}^{\prime}\right|$. Then

$$
A=\sup _{\nu>0}\left\{\left\|v_{\nu}\right\|_{L^{\infty}\left([0, T] \times \mathbb{R}^{2}\right)}\right\} \text { and } B=\sup _{\nu>0}\left\{\left\|v_{\nu}^{\prime}\right\|_{L^{\infty}\left([0, T] \times \mathbb{R}^{2}\right)}\right\}
$$

are finite by Theorem 1.5, so

$$
\sup _{\nu>0}\left\{\left\|f_{\nu}\right\|_{L^{\infty}\left([0, T] \times \mathbb{R}^{2}\right)}\right\} \leq(A+B)^{2}<\infty .
$$

Also by Theorem 1.5,

$$
\left\|g_{\nu}\right\|_{L^{p}\left(\mathbb{R}^{2}\right)} \leq C p\left\|\omega^{0}\right\|_{L^{p}\left(\mathbb{R}^{2}\right)} \leq \phi(p)=p(C \theta(p)),
$$

where $C \theta$ is an admissible function since $\theta$ is admissible by assumption. Applying Theorem 3.1 and Theorem 3.2 yields all three parts of Theorem 1.4 (only the uniqueness portions of parts (i) and (ii), though; see the comment following the statement of Theorem 1.4).

\section{Proof of Theorem 3.2}

In this section we prove Theorem 3.2 following the approach in [Y2].

Lemma 4.1. Let $D$ be a measurable subset of $\mathbb{R}^{n}$ and let $f$ and $g$ be nonnegative measurable real functions in $L^{1}(D) \cap L^{\infty}(D)$. Let $M \geq\|f\|_{L^{\infty}(D)}$. Assume that for some $p_{0}>1$ and some positive function $\phi:\left[p_{0}, \infty\right) \rightarrow \mathbb{R}^{+}$,

$$
\|g\|_{L^{p}(D)} \leq \phi(p)
$$

for all $p \geq p_{0}$. Then

$$
\int_{D} f(x) g(x) d x \leq \beta\left(\|f\|_{L^{1}(D)}\right) .
$$


Proof. Let $\epsilon$ be in $\left(0,1 / p_{0}\right]$. Then

$$
\begin{aligned}
\int_{D} f g & \leq M^{\epsilon} \int_{D} f^{1-\epsilon} g \leq M^{\epsilon}\left\|f^{1-\epsilon}\right\|_{L^{1 /(1-\epsilon)}}\|g\|_{L^{1 / \epsilon}} \\
& \leq M^{\epsilon}\|f\|_{L^{1}}^{1-\epsilon} \phi(1 / \epsilon)=\beta_{\epsilon}\left(\|f\|_{L^{1}}\right) .
\end{aligned}
$$

The conclusion follows from Equation (1.2).

Proof of Theorem 3.2. Letting $M=\sup _{\nu>0}\left\{\left\|f_{\nu}\right\|_{L^{\infty}\left([0, T] \times \mathbb{R}^{2}\right)}\right\}$ and $D=\mathbb{R}^{2}$ and applying Lemma 4.1, it follows that

$$
L_{\nu}(t)=\left\|f_{\nu}(t)\right\|_{L^{1}\left(\mathbb{R}^{2}\right)} \leq R \nu t+\int_{0}^{t} \beta\left(\left\|f_{\nu}(s)\right\|_{L^{1}\left(\mathbb{R}^{2}\right)}\right) d s,
$$

or,

$$
L_{\nu}(t) \leq R \nu t+\int_{0}^{t} \beta\left(L_{\nu}(s)\right) d s
$$

If $R=0$, Osgood's lemma immediately gives $L_{\nu} \equiv 0$. If $R>0$, we conclude that

$$
-\mathcal{M}\left(L_{\nu}(t)\right)+\mathcal{M}(R \nu t) \leq \int_{0}^{t} d s=t
$$

that is,

$$
\int_{R \nu t}^{L_{\nu}(t)} \frac{d s}{\beta(s)}=\int_{R \nu t}^{1} \frac{d s}{\beta(s)}-\int_{L_{\nu}(t)}^{1} \frac{d s}{\beta(s)} \leq t .
$$

It follows that for all $t$ in $(0, T]$,

$$
\int_{R \nu t}^{1} \frac{d s}{\beta(s)} \leq T+\int_{L_{\nu}(t)}^{1} \frac{d s}{\beta(s)} .
$$

As $t \rightarrow 0^{+}$, the left side of Equation (4.2) becomes infinite; hence, so must the right side. But this implies that $L_{\nu}(t) \rightarrow 0$ as $\nu \rightarrow 0^{+}$, and that the convergence is uniform over $[0, T]$.

\section{Rates of Convergence}

Define $f: \mathbb{R}^{+} \rightarrow \mathbb{R}^{+}$implicitly by

$$
\int_{x}^{f(x)} \frac{d s}{\beta(s)}=T .
$$

As $x$ decreases to zero, $f(x)$ monotonically decreases (to zero) because $\beta$ is positive. Also, because of Equation (4.1), $L_{\nu}(t) \leq f(R \nu t) \leq f(R \nu T)$, giving an expression for a uniform bound on the convergence rate. When $1 / \beta$ can be explicitly integrated, a bound on the rate can sometimes be determined in closed form. For the case of bounded vorticity, one obtains essentially the same bound on the rate as in [C2]. The sequence of bounds on vorticity in Equation (2.3) can also be handled this way, using the upper bound on the corresponding $\beta$ 
functions that Yudovich derives in [Y2]. In the notation of Section 2 this is $\beta(x)=x \psi(1 / x) \leq e x \theta_{m+1}(1 / x)$.

In general, though, one can bound the initial vorticity by an admissible function that will yield an arbitrarily slow bounded rate of convergence. This is because the function $f$, which was defined implicitly in terms of $\beta$, can, conversely, be used to define $\beta$, and we can choose $f$ so that it approaches zero arbitrarily slowly.

\section{Proof of Theorem 3.1}

In this section we establish Theorem 3.1, following Chemin's approach in [C2]. We consider three cases: 1) both $v_{\nu}$ and $v_{\nu}^{\prime}$ are solutions to $\left.\left(N S_{\nu}\right) ; 2\right) v_{\nu}$ is a solution to $\left(N S_{\nu}\right)$ while $v_{\nu}^{\prime}$ is a solution to $\left.(E) ; 3\right)$ both $v_{\nu}$ and $v_{\nu}^{\prime}$ are solutions to $(E)$.

Consider case 1. It follows from Theorem 1.5 that $v_{\nu}$ is in $L_{\text {loc }}^{\infty}\left(\mathbb{R} ; L^{p}\left(\mathbb{R}^{2}\right)\right)$ for all $p$ such that $2<p \leq \infty$; applying Hölder's inequality gives $v_{\nu} \cdot \nabla v_{\nu}$ in $L_{l o c}^{\infty}\left(\mathbb{R} ; L^{2}\left(\mathbb{R}^{2}\right)\right)$. An argument involving a Riesz transform (as in the proof of Yudovich's theorem in [C3], the extra viscosity term vanishing) then shows that $\nabla p_{\nu}$ is in this same space.

The assumption that $\nabla v_{\nu}$ is in $L_{l o c}^{\infty}\left(\mathbb{R} ; L^{2}\left(\mathbb{R}^{2}\right)\right)$ is enough to conclude via Theorem 1.5 that $w_{\nu}$ is in $L_{l o c}^{\infty}\left(\mathbb{R} ; W^{1,2}\left(\mathbb{R}^{2}\right)\right)$ and that $\Delta v_{\nu}$ is in $L_{l o c}^{\infty}\left(\mathbb{R} ; W^{-1,2}\left(\mathbb{R}^{2}\right)\right)$. It then follows from $\left(N S_{\nu}\right)$ that $\partial_{t} v_{\nu}$ is also in $L_{l o c}^{\infty}\left(\mathbb{R} ; W^{-1,2}\left(\mathbb{R}^{2}\right)\right)$. (For solutions to $(E)$, we reach the stronger conclusion that $\partial_{t} v_{\nu}$ is in $L_{\text {loc }}^{\infty}\left(\mathbb{R} ; L^{2}\left(\mathbb{R}^{2}\right)\right)$.)

Taking the inner product of both sides of the first equation in $\left(N S_{\nu}\right)$ with $w_{\nu}$ and subtracting the resulting equations for $v_{\nu}$ and $v_{\nu}^{\prime}$ gives

$$
\begin{aligned}
w_{\nu} \cdot \partial_{t} w_{\nu} & +w_{\nu} \cdot\left(v_{\nu} \cdot \nabla w_{\nu}\right) \\
& =-w_{\nu} \cdot \nabla\left(p_{\nu}-p_{\nu}^{\prime}\right)+\nu w_{\nu} \cdot \Delta w_{\nu}-w_{\nu} \cdot\left(w_{\nu} \cdot \nabla v_{\nu}^{\prime}\right) .
\end{aligned}
$$

Integrating both sides of Equation (6.1) over $[0, T] \times \mathbb{R}^{2}$, the pressure term disappears because $w_{\nu}$ is divergence-free. Similarly, the term $w_{\nu} \cdot\left(v_{\nu} \cdot \nabla w_{\nu}\right)$ disappears because $v_{\nu}$ is divergence-free, and we obtain

$$
\int_{0}^{T} \int_{\mathbb{R}^{2}} w_{\nu} \cdot \partial_{t} w_{\nu} d x d t=\int_{0}^{T} \int_{\mathbb{R}^{2}} \nu w_{\nu} \cdot \Delta w_{\nu}-w_{\nu} \cdot\left(w_{\nu} \cdot \nabla v_{\nu}^{\prime}\right) d x d t .
$$

But $w_{\nu}$ in $L_{\text {loc }}^{\infty}\left(\mathbb{R} ; W^{1,2}\left(\mathbb{R}^{2}\right)\right)$ and $\partial_{t} w_{\nu}$ in $L_{l o c}^{\infty}\left(\mathbb{R} ; W^{-1,2}\left(\mathbb{R}^{2}\right)\right)$ is sufficient to conclude (see, for instance, Lemma 1.2 p. 176 of $[\mathrm{T}]$ ) that

$$
\int_{0}^{T} \int_{\mathbb{R}^{2}} w_{\nu} \cdot \partial_{t} w_{\nu} d x d t=\frac{1}{2}\left\|w_{\nu}(T)\right\|_{L^{2}}^{2}
$$

where we have used $w_{\nu}(0)=0$.

It follows that

$$
\text { 1: }\left\|w_{\nu}(T)\right\|_{L^{2}}^{2}=2 \int_{0}^{T} \int_{\mathbb{R}^{2}} \nu w_{\nu} \cdot \Delta w_{\nu}-w_{\nu} \cdot\left(w_{\nu} \cdot \nabla v_{\nu}^{\prime}\right) d x d t .
$$


From the absolute continuity of the integral, we also conclude that $\left\|w_{\nu}(T)\right\|_{L^{2}}^{2}$ is an absolutely continuous function of $T$.

Following a similar procedure for the other two cases, we obtain

$$
\begin{aligned}
& \text { 2: }\left\|w_{\nu}(T)\right\|_{L^{2}}^{2}=2 \int_{0}^{T} \int_{\mathbb{R}^{2}} \nu w_{\nu} \cdot \Delta v_{\nu}-w_{\nu} \cdot\left(w_{\nu} \cdot \nabla v_{\nu}^{\prime}\right) d x d t \\
& \text { 3: }\left\|w_{\nu}(T)\right\|_{L^{2}}^{2}=-2 \int_{0}^{T} \int_{\mathbb{R}^{2}} w_{\nu} \cdot\left(w_{\nu} \cdot \nabla v_{\nu}^{\prime}\right) d x d t .
\end{aligned}
$$

For the term common to Equation (6.2)-Equation (6.4),

$$
\left|\int_{0}^{T} \int_{\mathbb{R}^{2}} w_{\nu} \cdot\left(w_{\nu} \cdot \nabla v_{\nu}^{\prime}\right) d x d t\right| \leq \int_{0}^{T} \int_{\mathbb{R}^{2}}\left|w_{\nu}\right|^{2}\left|\nabla v_{\nu}^{\prime}\right|^{2} d x d t .
$$

Since $w(t)$ is in $W^{1,2}\left(\mathbb{R}^{2}\right)$ for all time $t$,

$$
\int_{0}^{T} \int_{\mathbb{R}^{2}} w_{\nu} \cdot \Delta w_{\nu} d x d t=-\int_{0}^{T} \int_{\mathbb{R}^{2}}\left|\nabla w_{\nu}\right|^{2} d x d t \leq 0 .
$$

Similarly,

$$
\begin{aligned}
& \left|\int_{0}^{T} \int_{\mathbb{R}^{2}} w_{\nu} \cdot \Delta v_{\nu} d x d t\right| \\
& \quad \leq\left\|\nabla v_{\nu}\right\|_{L^{\infty}\left([0, T] ; L^{2}\left(\mathbb{R}^{2}\right)\right)}\left\|\nabla w_{\nu}\right\|_{L^{\infty}\left([0, T] ; L^{2}\left(\mathbb{R}^{2}\right)\right)} T \\
& \quad \leq C\left\|\omega^{0}\right\|_{L^{2}\left(\mathbb{R}^{2}\right)}^{2} T .
\end{aligned}
$$

Putting this all together gives Theorem 3.1 with, for the three cases,

$$
\text { 1: } R=0, \quad 2: \quad R=C\left\|\omega^{0}\right\|_{L^{2}\left(\mathbb{R}^{2}\right)}^{2}>0, \quad 3: \quad R=0 .
$$

In case 1, we only know that $R$, which comes from Equation (6.5), is negative or equal to 0 ; we cannot choose, a priori, a specific constant other than 0 .

If $v_{\nu}$ and $v_{\nu}^{\prime}$ were solutions for different initial conditions, then Equation (6.2) and Equation (6.4) would have the additional term $\left\|\omega_{\nu}(0)\right\|_{L^{2}}^{2}$ on the right-hand side. Modifying the argument in Section 2 to incorporate this term is the basis of the proof of existence in Theorem 1.4.

\section{Acknowledgement}

I wish to thank Misha Vishik for recommending that I read [C2] and [Y2] and combine the two results.

\section{References}

[C1] J.-Y Chemin, Sur le mouvement des particules d'un fluide parfait, incompressible, bidimensionnel, Inventiones Mathematicae, 103 (1991) 599-629.

[C2] J.-Y Chemin, A Remark on the Inviscid Limit for Two-Dimensional Incompressible Flu$i d s$, Commun. in Partial Differential Equations, 21 (11 \& 12), (1996) 1771-1779.

[C3] J.-Y Chemin, Perfect Incompressible Fluids, Oxford University Press, 1998.

[CW1] P. Constantin and J. Wu, Inviscid Limit for Vortex Patches, Nonlinearity 8 (1995), 735-742. 
[CW2] P. Constantin and J. Wu, The Inviscid Limit for Non-Smooth Vorticity, Indiana University Mathematics Journal, 45 (1996), 67-81.

[CW3] P. Constantin and J. Wu, Statistical solutions of the Navier-Stokes equations on the phase space of vorticity and the inviscid limit, J. Math Phys., 38, June (1997), 3031-3045.

[K] T. Kato, Remarks on zero viscosity limit for nonstationary Navier-Stokes flows with boundary, Seminar on nonlinear partial differential equations Berkeley, Calif., (1983), 8598.

[L] P. L. Lions, Mathematical Topics in Fluid Mechanics, Volume 1, Incompressible Models, Clarendon Press, Oxford, 1996.

[S] P. Serfati, Pertes de régularité pour le laplacien et l'équation d'Euler sur $\mathbb{R}^{n}$, preprint, 15 pp.

[T] R. Temam Navier-Stokes Equations: Theory and Numerical Analysis, American Mathematical Society, 1984, 2001.

[Y1] V. Yudovich, Nonstationary Flow of an Ideal Incompressible Liquid, Zhurn. Vych. Mat. 3 (1963), 1032-1066. (Russian)

[Y2] V. Yudovich, Uniqueness Theorem for the Basic Nonstationary Problem in the Dynamics of an Ideal Incompressible Fluid, Mathematical Research Letters, 2 (1995), 27-38.

Department of Mathematics, University of Texas, Austin, Texas, 78712

Current address: Department of Mathematics, University of Texas, Austin, Texas, 78712

E-mail address: kelliher@math.utexas.edu 